\title{
Experiencias de innovación en ámbitos públicos: "Legalización de Predios bajo tenencia de sus posesionarios"
}

\section{Innovation experiences in public spheres: "Land legalization under their owners tenure"}

\author{
Genesis Robles Zambrano $^{1 *}$, Juan Carlos Arandia Zambrano ${ }^{1}$ y Karen Clemencia Triviño Vera ${ }^{1}$ \\ ${ }^{1}$ Universidad de Quevedo \\ *genesisrobles@hotmail.com
}

DOI: https://doi.org/10.26871/killkana_social.v3i2.468

\begin{abstract}
Resumen
El presente trabajo realiza una valorización de las concepciones que tiene en la actualidad la vinculación con la sociedad y de qué manera se instituyó en el Ecuador por primera vez. Además analiza de manera crítica uno de los proyectos que se está desarrollando en la UNIANDES, denominado "Legalización de Predios Urbanos y Rurales que se encuentran bajo Tenencia de sus posesionarios". Se realizó investigación documental, aplicando métodos de análisis, síntesis e interpretación de contenidos. Se considera la Vinculación con la Sociedad como uno de los pilares misionales de la universidad en general, junto con la docencia y la investigación, ya que por su carácter de utilidad vienen dados en función de la multiplicidad de ambientes, contextos, dimensiones y condiciones del entorno. Finalmente se identifican las perspectivas a futuro de la vinculación dentro de los ámbitos públicos. El trabajo permite concluir que la vinculación deberá siempre ser considerada como un puente para la comunicación con la sociedad y no con tildes políticos, ya que debe establecerse una conciencia de cooperación en donde las instituciones públicas, las universidades y la comunidad construyan y den soluciones gracias a la convergencia del trabajo.
\end{abstract}

Palabras clave: Vinculación, Proyecto, Sociedad, ámbito público.

\begin{abstract}
This research work evaluates the perceptions currently linked to society, and the way in which they were instituted for the first time in Ecuador. Additionally, this work performs a critical analysis of one of the developing projects at UNIANDES, called "Legalization of Urban and Rural Lands under their owners' tenure. Documentary research was carried out through the application of methods such as analysis, synthesis, and content interpretation. Linkage with the Society, along with research and teaching, are considered the missionary bases of the university because of their usefulness. They respond to the multiplicity, dimensions, and environmental context conditions. Finally, the future perspectives of linkage within public spheres have been identified. This work leads to the conclusion that linkage should always be considered as a bridge that enables communication with society, and should not be influenced by political interests. Instead, an awareness of cooperation must be established so that the work convergence, public institutions, universities, and the community are able to build and give solutions to this issue
\end{abstract}

Key words: Linkage, Project, Society, Public spheres.

\section{Introducción}

La innovación social está experimentando desde hace más o menos una década un creciente interés en los sectores académicos, al tiempo que los gobiernos comienzan a integrarla en sus agendas políticas.

Razón por la cual se ha hecho primordial que los estudiantes se involucren con la sociedad en la resolución de conflictos que estén perfilados en la profesión que ejercerán, no solo llevándolo a la práctica o a las pasantías. Esto debe ejecutarse previo al proceso del cual la Educación Superior veíia acostumbrado.
Es de suma importancia para la materialización de un modelo educativo integral que los participantes se apropien del conocimiento en el contexto que se les coloque.

Para las instituciones educativas y los agentes de la función pública, la función social desde una perspectiva del servicio es una oportunidad de aprovechar los recursos intelectuales en acciones que sirvan para la formación integral de los estudiantes, con la atención en la asistencia, gestión y desarrollo, que propicien el bienestar social.

Es por ello que se hace primordial que los gobiernos municipales dejen a un lado la visión generalista de los pro- 
blemas ya que las instituciones universitarias constituyen una instancia estratégica como potenciadora de sinergias para el desarrollo local y regional.

Legalización de Predios Urbanos y Rurales que se encuentran bajo Tenencia de sus posesionario

La teoría y la praxis sobre la innovación social ha ido creciendo a pasos agigantados. Al mismo tiempo, muchas necesidades, demandas y expectativas de los ciudadanos no se abordan eficazmente por parte de los poderes públicos, en un contexto de creciente desafección ciudadana y déficit de legitimad del sistema político democrático. Por tanto, los desafíos sociales exigen una nueva perspectiva en el análisis y la aplicación de la innovación.

En base a lo antes expuesto, se puede deducir que la innovación social es vista como una forma de compensar las falencias del estado, o mejor dicho, de los poderes públicos, a pequeños o grandes problemas sociales que de algún modo pueden ser resueltos por otros agentes sociales, como lo son las universidades e institutos universitarios.

Ahora bien, uno de los mecanismos para promover que la juventud estudiantil participe en el desarrollo del Ecuador, es potencializando la vinculación de las necesidades económicas y sociales de cada región con proyectos educativos que den soluciones a problemas concretos.

Una de las actividades que han sido incorporadas en el sistema de la universidad ecuatoriana es la Vinculación con la colectividad, la cual es definida como una de las tareas primordiales, y lo que se busca fehacientemente es implementar un cambio social duradero; donde se involucre a los estudiantes con la comunidad, haciendo que vivan y sientan empatía con esta. Es una de las maneras de generar preocupación por las personas y la sociedad en conjunto ya que de esta forma una vez creado el vínculo, se sientan en la necesidad de mejorar las vidas de los demás, dándole solución a un problema que ellos mismos palpen.

La figura de vinculación con la sociedad se manifiesta en la Educación Superior del Ecuador en el año 2002 con el CONEA, quien la difundió con el afán de establecer las acciones que ejecutarían las universidades e Institutos de Educación Superior con relación a la comunidad.

Es importante preguntarse: ¿qué es realmente esta actividad y por qué tiene cada vez más ponderación en la educación?, según el profesor Giacomo Gould Bei el modelo educativo entiende la vinculación como "el proceso integral que articula las funciones sustantivas de docencia, investigación, y extensión de la cultura". A partir de dicha definición, la vinculación es un proceso que involucra no solo a la universidad con la comunidad sino al sistema educativo en su mayor expresión con la sociedad.

Desde la perspectiva latinoamericana algunos autores como Leopoldo Zea, consideran que las universidades tienen un compromiso con la sociedad, ya que es agente de cambio capaz de generar los conocimientos teóricos y prácticos que elevan la creación y recreación de sus individuos, necesarios para la solución de problemas a través de proyectos que estimulen la interacción recíproca universidad-comunidad, lo cual permite desarrollar la vida nacional en todos sus aspectos.

Por su parte, Malagón (2006) considera que el conocimiento debe ser apropiado de diferentes maneras, dentro y fuera de la universidad, ya que reconoce que la producción del conocimiento constituye una de las formas más claras de interacción entre la universidad y el contexto en que se inserta.

Entonces se puede entender que el objetivo de las vinculaciones de los estudiantes con la sociedad, no solo se realiza con el fin que los conocimientos no se queden en las aulas de clases, sino que puedan aportar lo que se aprende y ponerlo en práctica para resolver problemas que puedan verse en las comunidades. En circunstancias anteriores había que esperar hasta las prácticas profesionales o las pasantías para que los estudiantes universitarios tuvieran un contacto directo con la realidad de la profesión y/o carrera que cursaban, toda esta realidad ha cambiado.

Por otro lado, se evidencia que el proceso de formación del alumnado por medio de las actividades de vinculación tiene relación con el modo de producción del conocimiento que muchas veces complementa un modelo investigativo universitario. Se muestra cómo al estudiante no le basta con lo aprendido en las instituciones educativas sino que investiga, explora, indaga nuevas opciones para dar soluciones a la comunidad con la cual se le involucra.

De modo que, la presencia de la pretensión de ser útil a la comunidad, fortalece la participación en un proceso donde el conocimiento y sus resultados se difundan a la sociedad a través de las habilidades y experiencias que aportan los que intervienen en ella. Los aprendizajes estarán dados en función de la multiplicidad de ambientes, contextos, dimensiones y condiciones del entorno.

Indiscutiblemente desde esta óptica, la Vinculación con la Sociedad podría considerarse como uno de los pilares misionales de la universidad en general, junto con la docencia y la investigación.

En este sentido se hace indispensable y necesaria la colaboración de las municipalidades. Es primordial que los gobiernos municipales dejen a un lado la visión generalista de los problemas, ya que las instituciones universitarias constituyen una instancia estratégica como potenciadora de sinergias para el desarrollo local y regional.

La Universidad de los Andes (UNIANDES), cuenta con varios convenios firmados con los Gobiernos Autónomos Descentralizados (GAD), con la finalidad de llevar a cabo proyectos que son elaborados por los docentes con la participación activa de los estudiantes.

Amplia es la gama de proyectos que se han llevado de la mano con el apoyo y la colaboración del sector público. En la actualidad se desarrolla un proyecto en vista de una necesidad evidente, y con el aprovechamieno de la pertinencia con la carrera de derecho, se quiere ayudar y asesorar a las comunidades de los sectores rurales de la región que no contaban con escrituras ni títulos de propiedad de sus 
terrenos a tener acceso a través de los estudiantes a trámites administrativos.

El Gobierno Autónomo Desentralizado (GAD) de Quevedo y Registro de la Propiedad ubicados en la Cdla. Municipal de la Parroquia Nicolás Infante Díaz, del cantón Quevedo de la provincia de Los Ríos amparados en el Convenio de cooperación con UNIANDES y ante la necesidad de determinar y solucionar el problema que atañe a la población rural, se elaboró un proyecto denominado Legalización de Predios Urbanos y Rurales que se encuentran bajo Tenencia de sus posesionarios.

El proyecto se ha desarrollado con el involucramiento del equipo transdisciplinario, integrado por el Departamento de Avalúos y Catastros, legalización de terrenos del GAD y el Registro de la Propiedad del cantón en cuestión.

Para la realización y/o elaboración del proyecto se vio la necesidad de:

- Determinar los sectores y los habitantes que mantienen problemas de legalización por desconocimiento procedimental.

- Realizar un levantamiento estadístico documental que certifique la posesión del bien inmueble.

- Asesorar a los habitantes del cantón, para que accedan a los beneficios de la obtención de los títulos de propiedad.

En este sentido, es importante destacar que la eficacia de este pilar misional como se le denominó, está en la solución de problemas en los sectores comunitarios específicos, en la aplicación, intercambio, y búsqueda de nuevos conocimientos para ayudar a esa comunidad con el apoyo de las entidades públicas a resolver problemas sociales, y aprovechar al máximo la pertinencia con cada profesión.

Pero no se puede dejar a un lado un factor de suma importancia como lo es el impacto social. Este juega un papel con gran ponderación para la sostenibilidad de cualquier proyecto de vinculación, y para la progresividad en la elaboración y aceptación de futuras propuestas de proyectos dentro del sector público. No solo debe ser confiable y cumplir con las metas propuestas al momento de su elaboración sino que debe sostenerse y poder ser constatado por las entidades públicas, ya que no solo están en tela de juicio la reputación y credibilidad de las instituciones universitarias sino también las entidades públicas.

Es por ello que se esperan tener unos resultados cuantificables, correlacionados con las necesidades ya identificadas, como lo es la identificación del área de ubicación de los terrenos y los habitantes que mantienen la posesión, la obtención de documentación que acredita la posesión del bien inmueble para que los estudiantes puedan llevar a cabo el trámite administrativo antes las entidades públicas competentes, y por último llevarlos a la adquisición del título de propiedad del inmueble del que son posesionarios.

Hay que destacar que el árbol del problema precisará los aspectos más relevantes para la intervención en la legalización de los terrenos urbanos y rurales, que en este caso no solo se llevará a cabo en la Parroquia San Carlos del cantón Quevedo sino también evaluando la necesidad de la zona, se extiende al cantón Valencia Nueva Unión Etapa 2, en donde han sido asignados dos grupos de estudiantes de la casa de estudio UNIANDES.

Se identificaron como causas primarias de la problemática la ausencia de información y desconocimiento en la tramitación de los requisitos para adquirir la legalización del bien y que al no ser correctamente asesorados, serían la razón principal de que los habitantes mantengan su estado de posesionarios fungiendo ser dueños de la propiedad, cuando para efectos legales deben ser propietarios del bien y no solo meros posesionarios.

En este sentido se realizó una descripción del problema que puede ser representada de la siguiente manera:

1) Los poseedores de los predios confunden la mera tenencia de la posesión, con propiedad, lo que demuestra la conformidad de la comunidad ante esta situación legal.

2) Desaprovechan de las ventajas y beneficios de ser propietarios, al desconocer los beneficios que puede traer consigo ser titular de una propiedad, no muestran interés en préstamos de mejoras que puedan generar mejores condiciones de vida.

3) Ser posesionarios crea condiciones inconformes de urbanismo, vivienda y calidad de vida, esta causa esta enlazada con la anterior.

4) Existe falta de acceso a la información acerca del procedimiento para la obtención del título de propiedad del bien inmueble, ya que muchas veces se encuentran en zonas tan rezagadas impidiendo el acceso a la asesoría jurídica-legal lo que en realidad dificulta conocer que el trámite en la mayoría de los casos puede ser sencilla.

5) Se evidencia un desconocimiento de la normativa legal que les permite obtener el título de propiedad, muchas veces la gran mayoría de estos poseedores desconocen los beneficios legales que les permiten obtener el título del inmueble, ya que tienen miedo de desalojo cuando en realidad pueden tramitar sus escrituras por el tiempo que tienen habitando los predios.

Es menester destacar que el proyecto, fue propuesto en correspondencia al objetivo $\mathrm{N}^{\circ} 1$ del Plan Nacional del Buen vivir 2017-2021, que a su vez manifiesta: Garantizar una vida digna con iguales oportunidades para todas las personas; concatenado con la política 1.8 que establece la garantía del acceso a una vivienda adecuada y digna, con pertinencia cultural y a un entorno seguro, que incluya la provisión y calidad de los bienes y servicios públicos vinculados al hábitat: suelo, energía, movilidad, transporte, agua y saneamiento, calidad ambiental, espacio público seguro y recreación.

Al acercar a los estudiantes a esta realidad del sector, se siente el compromiso, no solo de estos sino de las entidades públicas involucradas de la mano con las casas de estudio, lo que de alguna manera les garantiza a los habitantes de los sectores menos favorecidos el cumplimiento de los proyectos. 
En este punto se llega a un reto, aun cuando el proyecto se evalúa y se mide por sus impactos sociales, ambientales, por la pertinencia, surgen las siguientes interrogantes: ¿Qué sucede si el proyecto no se cumple en el tiempo propuesto o si no se cumplen las metas que se tenían planteadas?, ese impacto social se puede revertir, y ser perjudicial, tanto para la comunidad como para las entidades públicas.

Hay que considerar que el impacto social puede darse a través de la innovación social, y que debe concebirse como un instrumento para incidir sobre las causas de la problemática, para reducir la exclusión y la discriminación social, y que en el proceso pueda darse una actuación o iniciativa que responda de modo efectivo a este reto que debe cumplir ciertos criterios mínimos que aseguren un desarrollo coherente del proyecto.

En este sentido, la Universidad Regional Autónoma de los Andes, durante su trayectoria ha dado muestra del compromiso social, en concreto en el servicio a la sociedad a través de la educación de tercer nivel y que en la actualidad fortalece esta visión con proyectos que benefician a la sociedad.

Ahora bien, se puede decir que el proyecto apoya la disminución de la falta de legalización de terrenos urbanos y rurales que se encuentran como mera tenencia de sus posesionarios en los sectores vulnerables, y al logro de los objetivos del Plan de Ordenamiento Territorial del cantón Quevedo y Valencia de la provincia de los Ríos, que contribuyen al logro de los objetivos del buen vivir.

\section{Metodología}

El presente trabajo se realizó a través de una investigación documental, según Alfonso (1995), como un procedimiento científico, un proceso sistemático de indagación, recolección, organización, análisis e interpretación de información o datos en torno a un determinado tema.

Por otro lado, en la presente investigación se utilizó el método deductivo ya que se parte los datos generales aceptados como valederos, para deducir por medio del razonamiento lógico, varias suposiciones, es decir; parte de verdades previamente establecidas como principios generales, para luego aplicarlo a casos individuales y comprobar así su validez.

Cabe destacar que el razonamiento deductivo constituye una de las principales características del proceso de enfoque cuantitativo de la investigación, por tanto la modalidad en la cual se enmarca el presente trabajo es cualitativa.

\section{Conclusiones}

La vinculación con la sociedad deberá siempre ser considerada como un puente para la comunicación con la sociedad y no con tildes políticos, ya que debe establecerse una conciencia de cooperación en donde las instituciones públicas, las universidades y la comunidad construyan y den soluciones gracias a la convergencia del trabajo.
Los estudiantes intervienen en una realidad que requiere mejorarse y se convierte en la extraordinaria oportunidad para que confronten dialógicamente los conocimientos teóricos con la práctica, con el desarrollo de competencias integrales, habilidades, destrezas, compromisos y competencias; descubriendo aptitudes que por falta de oportunidad, no se evidencian. El proyecto como recurso didáctico ha consolidado el pensamiento crítico - analítico y el compromiso social.

El proyecto genera un impacto social cuantificable, ya que los beneficiarios del mismo obtendrán la titularidad de la propiedad.

\section{Recomendaciones}

Los bienes inmuebles deben registrarse por sus propietarios o posesionarios, ante el seguimiento del trámite administrativo por parte de los estudiantes, cuya necesidad radica en el derecho que les asiste a todos y cada uno de los habitantes de garantizar la tenencia de dichos terrenos.

Los estudiantes, para poder asesorar legalmente a los habitantes de la comunidad, deberán aplicar los conocimientos obtenidos durante el estudio de la carrera de Derecho, por tanto es indispensable la participación activa de los mismos en el desarrollo del proyecto.

No es necesario que los participantes estén en los últimos niveles de la carrera, ya que de esta manera se puede incentivar y promover la investigación de campo por así denominarla a descubrir nuevos conocimientos no estudiados para darle solución a la problemática presentada.

Limitar la participación de los entes públicos cuando puedan tomar tildes políticos en el desarrollo de los proyectos, de esta manera se genera confianza en la comunidad.

\section{Referencias Bibliográficas}

Giacomo Gould, B. (2002). La administración de la vinculación: cómo hacer qué. México, D.F: Comisión Nacional de Libros de Texto Gratuitos.

Malagón-Plata, L. (2006). La pertinencia curricular un estudio en tres programas universitarios educación y educadores. Cundinamarca, 12. Descargado de https://www.redalyc.org/pdf/ $834 / 83411512003$.pdf

Naishtat, F. (1998). Autonomía académica y pertinencia social de la universidad pública: una mirada desde la filosofía política. world congress of philosophy. Descargado de http: / / www.bu.edu/ wcp/Papers/Educ/EducNais.htm (Boston)

Sepúlveda, L., y Valdebenito, M. J. (2014). Aspiraciones y proyectos de futuro de estudiantes de enseñanza técnica-profesional. Polis Revista Latinoamericana. Descargado de http: / / journals . openedition.org/polis/10269

Zea, L. (2006). Estudios de filosofía práctica e historia de las ideas. Scielo. 
Recibido: 12 de mayo de 2019

Aceptado: 16 de julio de 2019

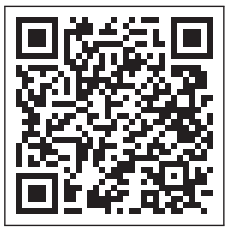


